Introduction: Machine learning has increasingly been applied to classification of psychosis spectrum in neuroimaging research. However, a number of multimodal studies using MRI and electroencephalography (EEG) is quite limited.

Objectives: To assess the power of multimodal structural MRI (sMRI) and EEG data to provide pairwise discrimination between first-episode schizophrenia (FES) patients, individuals at ultrahigh-risk of psychosis (UHR), and healthy controls (HC) using machine learning algorithms.

Methods: 46 FES male patients, 39 UHR individuals, and 54 matched HC underwent sMRI (3T Philips scanner) and electroencephalography. T1-weighted images were processed via FreeSurfer to obtain cortical and subcortical measures. L2 regularized logistic regression was used to evaluate the efficacy of diagnostic prediction.

Results: The accuracies of pairwise discriminations were: $87 \%$ for FES vs HC (specificity $83 \%$, sensitivity $91 \%$ ); $77 \%$ for FES vs UHR (specificity $76 \%$, sensitivity $79 \%$ ); $75 \%$ for UHR vs HC (specificity $77 \%$, sensitivity $73 \%$ ).

Conclusions: Current findings suggest that the patterns of anatomical and functional variability have potential as biomarkers for discrimination between schizophrenia, UHR, and healthy subjects. Furthermore, results show that the selection and multimodality of feature types are important. Specifically, adding EEG data to morphometric measures improved accuracy rates in FES vs HC and FES vs UHR contrasts, whereas standalone EEG data provided higher accuracy compared with morphometric or multimodal data in UHR vs HC discrimination. Expectedly, predictive power for the UHR was smaller than for the FES due to its intermediate anatomical features, located between those observed in healthy controls and those found in patients. The work was supported by RFBR grant 20-013-00748

Keywords: machine learning; psychosis; MRI; EEG

\section{Neuroscience in psychiatry}

\section{EPP0804}

Gastrointestinal functional impairments and epilepsy: Searching the possible connection mechanisms

\author{
R. Lefter ${ }^{1}$, A. Ciobica ${ }^{1,2,3}$ and I.M. Balmus ${ }^{4 \star}$ \\ ${ }^{1}$ Center Of Biomedical Research, Romanian Academy, Iasi, Romania; \\ ${ }^{2}$ Department Of Biology, Faculty Of Biology, Alexandru Ioan Cuza \\ University of Iasi, Iasi, Romania; ${ }^{3}$ Department Of Biology, Academy of \\ Romanian Scientists, Bucharest, Romania and ${ }^{4}$ Department Of \\ Interdisciplinary Research In Science, Alexandru Ioan Cuza University \\ of Iasi, Iasi, Romania \\ ${ }^{*}$ Corresponding author. \\ doi: 10.1192/j.eurpsy.2021.1098
}

Introduction: Epilepsy is one of the most common neurological disorders worldwide characterized by unpredictable and recurrent seizures, resulting from abnormal brain activity, accompanied by loss of consciousness and control of bowel or bladder function. Objectives: A higher risk of comorbid disorders in epilepsy has been reported for psychiatric affective conditions (i.e., depression and schizophrenia), sleep alterations, as well as some gastrointestinal disorders (inflammatory bowel disease and constipation), and lately there is an interest to determine and explain a putative association between functional gastrointestinal disorders (FGID) such as Irritable bowel syndrome (IBS) and epilepsy.

Methods: In this way, we decided to review the current aspects of the gastrointestinal functional impairments and epilepsy by searching in the literature possible connection mechanisms.

Results: A handful of studies have only recently reported an increased prevalence of IBS in epilepsy in children, in adults, and conversely a higher incidence of epilepsy in IBS patients at the populational level. Paroxysmal abdominal complaints resulting from seizure activity are present in the abdominal epilepsy syndrome and the link between constipation and seizures has been demonstrated in animal models. Currently, there is no data to directly address the cellular and molecular connections between epilepsy and FGID, but these would probably involve the bidirectional dysregulation of the brain-gut axis with increased afferent processing of visceral nociceptive signals and subsequent hyperalgesia.

Conclusions: Thus, intestinal dysbiosis may play a role in triggering inflammatory and immune-related mechanisms reported in IBS manifestations and epilepsy, while vagal neuroimunomodulation issues are likely to be involved in both pathologies as well.

Conflict of interest: The authors are currently supported by a Young Research Teams supporting research grant PN-III-P1-1.1TE2016-1210, named "Complex study on oxidative stress status, inflammatory processes and neurological manifestations correlations in irritable bowel synd

Keywords: FGID; Epilepsy; irritable bowel syndrome; mechanisms

\section{EPP0805}

\section{Affective and cognitive impairments in patients with} epilepsy

I. Blazhina ${ }^{1}$ and V. Korostiy ${ }^{2 *}$

${ }^{1}$ Department Of Nervous Diseases Psychiatry And Medical Psychology, Bucovinian State Medical University, Chernivtsi, Ukraine and ${ }^{2}$ Psychiatry, Narcology, Medical Psychology And Social Work, Kharkiv National Medical University, Kharkiv, Ukraine

${ }^{\star}$ Corresponding author.

doi: 10.1192/j.eurpsy.2021.1099

Introduction: The most common psychiatric conditions in epilepsy are depression, anxiety, behavioral, psychotic disorders and cognitive disorders as well as those which can be caused by convulsive seizures.

Objectives: The aims of the research were to define cognitive and affective impairments in patients with epilepsy and their quality of life. Since the presents of cognitive impairments and affective disorders have a considerable impact on the functioning of patients, their socialization and the level of their disability.

Methods: We studied the features of clinical and psychopathological manifestations in patients suffering from epilepsy. The study covered 100 patients ( 47 men and 53 women) who were in inpatient care. The following psychodiagnostic techniques were used: the test of 10 words of Luria, the MOCA test, the Münsterberg test, MiniMult test, the quality of life scale, the Hamilton scale of depression and anxiety.

Results: The following data of the study were observed: $88 \%$ patients had decreased memory, $38 \%$ had symptoms of depression, $28 \%$ had mild situational or neurotic depression, $8 \%$ had moderate depression, $2 \%$ had severe depression, $20 \%$ had a state of severe 